\title{
Development of an effective interatomic potential for transition metals and alloys: Modified Wills-Harrison model
}

\author{
S RAJU, E MOHANDAS and V S RAGHUNATHAN* \\ Metallurgy Division, Indira Gandhi Centre for Atomic Research, Kalpakkam 603 102, India
}

\begin{abstract}
This paper deals with the development of an effective interionic interaction for transition metals and alloys in the spirit of a hybridized pseudopotential tight-binding formalism, developed among others by Wills and Harrison. Stated briefly, the recipe consists in writing the total interionic interaction as a sum of contributions arising from nearly free s-electrons and that of tightly bound d-electrons. The s-d hybridization is also accounted for by allowing for the relative change in the occupancy of $s$ and d electron counts. This potential, although not taking into account the magnetic contribution, contains otherwise all the essentials of a physically meaningful interatomic interaction. In this paper, a modified version of the Wills-Harrison model is used to calculate the structural and cohesive properties of transition metals at $0 \mathrm{~K}$. The calculated values are in reasonable agreement with the experimental ones. This method is also used to derive an effective interatomic interaction for $\gamma$-TiAl. The use of this potential in obtaining an estimate of point defect energetics in TiAl is discussed.
\end{abstract}

Keywords. Interatomic potentials; Wills-Harrison model; TiAl; vacancy properties.

\section{Introduction}

The availability of simple and physically appealing interatomic potentials is rather crucial for meaningful modelling studies of condensed systems (Gehlen et al 1972). In the realm of physical metallurgy, the use of empirical and semi-empirical pair potentials has a long history in that diverse issues ranging from alloy phase stability to defect structure modelling have been tackled using the so-called fitted potentials (Gehlen et al 1972; deHosson 1981). In spite of easy applicability, the empirical potentials lack a strong theoretical basis, making thereby the results obtained from them rather difficult to be interpreted (Carisson 1990). In brief, it may be said that empirical interatomic potentials are neither transparent nor transferable (deHosson 1981; Carlsson 1990).

\section{Wills-Harrison model}

\subsection{Genesis}

In the realm of metal physics, the use of pseudopotential theory has provided a simple and neat picture of simple metal cohesion (Harrison 1970). The effective interionic interaction in this case may be approximated (Harrison and Wills 1982) to be consisting of a net Coulombic repulsion, modified by a screening factor. It follows that to a first-order approximation, no explicit introduction of a structure-dependent term is necessary to account for the bulk properties (Harrison 1970; Harrison and Wills 1982). Thus the failure to hold the Cauchy relation $\left(\mathrm{C}_{12}=\mathrm{C}_{44}\right)$ is directly explained from the volume-dependent repulsive ion-ion interaction. Of course, it is necessary and

*Author for correspondence 
desirable to include the structure-dependent term when it comes to calculating, say the relative structural stabilities (Hafner 1987). But this point does not concern us here. On the other hand, it was generally ascribed that owing to strong scattering $d$-electron potential a perturbative treatment of the total energy of transition metals is impractical (Ashcroft and Mermin 1976). But the crux of the point is that if one could separate some how the free electron like s-state from the deeply bound $d$-state and describe their coupling as a perturbation in an appropriate expandable parameter (Harrison and Froyen 1980), meaningful and useful simplifications of the transition metal band structure can still be achieved on lines similar to the development of pseudopotential formalism (Wills and Harrison 1983). Starting from early seventies to the present time, the torrent of developments in the theory of transition metal cohesion has taken the field far beyond the aforementioned simple description. The readers are referred to the reviews (Harrison 1980; deHosson 1981, 1989; Pettifor 1983) for further details. However, it is sufficient to mention that the genesis of the Wills-Harrison Model (WHM) (Wills and Harrison 1983) is strongly rooted in the multifarious developments concerning the electronic structure of transition metals. In this paper a modified version of the original WHM is adopted to probe a few structural metallurgical issues. A brief account of the basics of WHM is presented below.

\subsection{Basic description}

The total potential energy of a condensed system consisting of ions (nucleus + core electrons) and the outer valence electrons is generally given by (Gehlen et al 1972)

$$
\phi=\phi_{s}(V)+\frac{1}{2} \sum \dot{V}_{\mathrm{eff}}(r ; V),
$$

where, the summation is carried over to all $\left\{r_{i}\right\}$, the set of lattice positions. The first term in RHS is basically a many body effective ion-ion interaction term. In WHM (Wills and Harrison 1983), the $s$ - and $p$-electron contributions to cohesion are treated in a nearly free electron pseudopotential. The contribution from the $d$-band broadening and $s-d$ hybridization are treated in the framework of linear muffin tin orbital (LMTO) formalism. By superposing these two contributions to the total energy, an effective real space ion-ion potential is derived in a form, which is similar to (1). The $V_{\text {eff }}$ may further be explicitly split into two contributions as given by

$$
V_{\text {eff }}(r)=V_{s}(r)+V_{d}(r)
$$

where $V_{s}(r)$ is the nearly free electron contribution and is given by (Harrison and Wills 1982)

$$
V_{s}(r)=\frac{\left(Z_{s} e\right)^{2}}{r} \cos h^{2}\left(\kappa r_{\mathrm{c}}\right) e^{-\kappa r_{c}},
$$

where $\kappa^{2}=4 k_{f} m e^{2} / \hbar^{2} \pi$ is the square of the Thomas-Fermi screening length, $r_{\mathrm{c}}$ the Ashcroft's empty core pseudopotential radius and $Z_{s}$ the number of valence s-electrons. This potential is derived using the Thomas-Fermi linear screening approximation (Harrison 1970; Harrison and Wills 1982). The purely repulsive form of $V_{s}(r)$ derives from this assumption. On the other hand, if one employs a Lindhard approximation for the Hartree dielectric function, a potential with a long-range oscillatory behaviour is obtained (Harrison 1970). 
The effective $d$-band contribution $\left(V_{d}(r)\right)$ is obtained by coupling $d$-orbitals on different sites with the conduction s-electrons. In WHM, this is evaluated in the spirit of LMTO theory and Hartree-Fock free atom wave functions. The tight-binding matrix elements are represented in this model by a quantity $r_{d}$ called the $d$-orbital radius of the free atom. Thus, we get

$$
V_{d}(r)=V_{d}^{b}(r)+V_{d}^{r}(r),
$$

where in $V_{d}^{b}(r)$, the bonding $d$-contribution is given within the framework of Friedel's rectangular density of $d$-states model as (Wills and Harrison 1983)

$$
V_{d}^{b}(r)=-Z_{d}\left(1-Z_{d} / 10\right) \sqrt{12 / n}(28 \cdot 1 / \pi) h^{2} r_{d}^{3} / m r^{5},
$$

where $n$ is the first coordination number. The repulsive $V_{d}^{r}(r)$ due to the hybridization shift in the centre of gravity of the $d$-states is given by (Wills and Harrison 1983)

$$
V_{d}^{r}(r)=-Z_{d}\left(225 / \pi^{2}\right) \hbar^{2} r_{d}^{6} / m r^{8},
$$

where $Z_{d}$ is the number of bonding $d$-electrons. Barring $Z_{s}, Z_{d}, r_{\mathrm{c}}, n$ and $r_{d}$, there are no other adjustable parameters. In fact all these values are available from standard sources (Harrison 1980; Harrison and Froyen 1980; Wills and Harrison 1983). Further $r_{c}$ can also be determined using the familiar zero pressure condition $\left((\partial E / \partial V)_{V_{\text {eq }}}=-p=0\right)$. In table 1 , the input parameters needed for calculating $V_{\text {eff }}(r)$ are presented for selected transition metals.

\subsection{Modified Wills-Harrison scheme}

The values of bulk modulus at zero pressure $\left(B_{0}\right)$ calculated vide $(1)-(6)$, show rather poor agreement with the experimental values. This is partly due to the neglect of magnetic interactions in late $3 d$ metals and spin-orbit coupling in heavier transition metals. Since a rigorous description of both of these is beyond the scope of the original WHM, we choose to modify the WHM prescription in an ad hoc manner by incorporating twe additional parameters, $k_{1}$ and $k_{2}$ such that

$$
V_{\text {eff }}(r)=V_{s}(r)+k_{1} V_{d}^{b}(r)+k_{2} V_{d}^{r}(r)
$$

The constants $k_{1}$ and $k_{2}$ are determined by requiring $V_{\text {eff }}(r)$ to simultaneously satisfy $B_{0}$ and $B_{0}^{\prime}$, the pressure derivative of bulk modulus at equilibrium volume. $B_{0}^{\prime}$ is given by

$$
B_{0}^{\prime}=\frac{1}{B_{0}}(-V \mathrm{~d} B / \mathrm{d} V)_{V=V_{0}} \text {. }
$$

The values of $k_{1}$ and $k_{2}$ obtained are listed in table 1 .

Table 1. List of input parameters.

\begin{tabular}{lccccccccc}
\hline Metal & $Z_{s}$ & $Z_{d}$ & $n$ & $r_{c}$ & $r_{d}$ & $r_{0}$ & $B_{0}(\exp )$ & $k_{1}$ & $k_{2}$ \\
\hline $\mathrm{Ti}$ & 1.5 & 2.5 & 12 & 0.91 & 1.08 & 1.61 & 1051 & 2.07 & 4.85 \\
$\mathrm{~V}$ & 1.5 & 3.5 & 8 & 0.87 & 0.98 & 1.49 & 1619 & 2.22 & 5.53 \\
$\mathrm{Cr}$ & 1.5 & 4.5 & 8 & 0.82 & 0.9 & 1.42 & 1901 & 2.15 & 5.04 \\
$\mathrm{Mn}$ & 1.5 & 5.5 & $8(\alpha-\mathrm{Mn})$ & 0.78 & 0.86 & 1.43 & 596 & 0.657 & 0.632 \\
$\mathrm{Fe}$ & 1.5 & 6.5 & 8 & 0.71 & 0.8 & 1.41 & 1683 & 2.274 & 4.96 \\
\hline
\end{tabular}

Note: all $r$ values are in $10^{-10} \mathrm{~m} . B_{0}$, the experimental bulk modulus values are in $10^{2} \mathrm{MPa}$. 

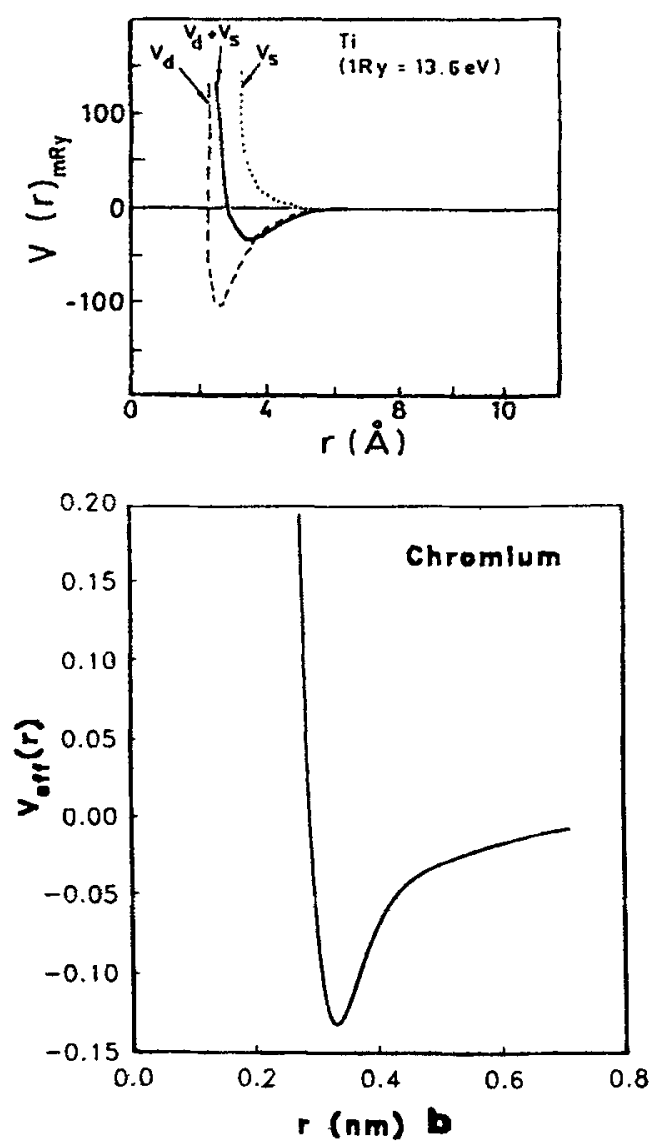

Figure 1. a. Calculated interatomic potential for Ti. Note the cancellation of strong repulsive contribution by the attractive d-band bonding contribution and $b$. calculated interatomic potential for $\mathrm{Cr}$.

\section{Discussion}

\subsection{General}

The calculated potential for titanium taken as a typical example is given in figure 1a. It can be seen that the strong repulsive $V_{s}(r)$ contribution is more than compensated by the $V_{d}^{b}$ term. It is also instructive to note that the minimum in $V_{\text {eff }}(r)$ occurs slightly to the right of the actual nn separation. In figure $1 \mathrm{~b}$ the calculated $V_{\text {eff }}(r)$ for chromium is given where in the experimental nn distance and the potential minimum again do not coincide.

This modified WHM potential has been used to calculate the Debye temperature of transition metals (Raju et al 1996a). It is sufficient to remark that the calculated values are in reasonable agreement with those obtained by more elaborate scheme (Haglund et al 1993).

Regarding its physical content, WHM offers interesting possibilities. In the first it is possible to obtain physically more complete functional forms for $V_{\text {eff }}(r)$ by employing better screening scheme and pseudopotentials. Such a potential is actually called for in 


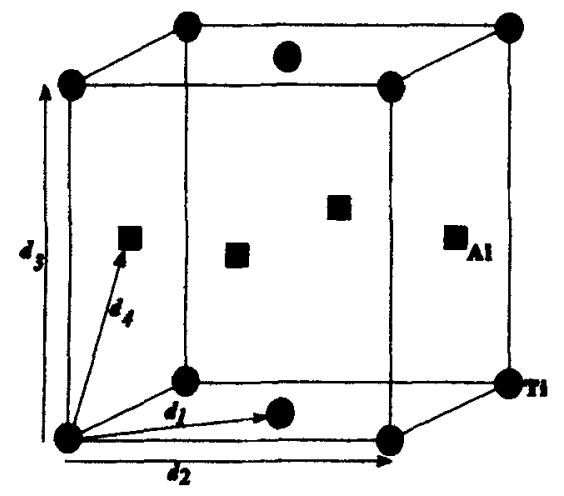

Figure 2. $L 1_{0}$ crystal structure of $\gamma$-TiAl. Different bond lengths are marked. Note $c / a=d_{3} / d_{2}>1$.

calculating the relative stabilities among competing crystal structures. Secondly, one can also use the valence partitioning into $Z_{s}$ and $Z_{d}$ as an adroitly adjustable tool to obtain optimized $V_{\text {eff }}(r)$, which is appropriate to a given crystal chemical environment (Raju et al, unpublished). Setting aside further discussion, we may briefly concentrate on the application of this potential to $\gamma$-TiAl intermetallic (Raju et al 1996b).

\subsection{Application to $\gamma$-TiAl}

$\gamma$-TiAl is a layered intermetallic with fct structure $(c / a \sim 1 \cdot 02$; figure 2$)$. This intermetallic has immense potential as a candidate material for high temperature aerospace applications and has been at the centre of intense research activities. In the present case we are interested in getting a first-order estimate of the point-defect properties by employing the WHM potential in conjunction with the quasi-chemical description of the defect equilibrium. A detailed account of this work has been published elsewhere (Raju et al 1996b).

Briefly the work involves calculating the $V_{\text {eff }}(r)$ for $\mathrm{Ti}, \mathrm{Al}$ and $\mathrm{Ti}-\mathrm{Al}$ interactions with reference to the alloyed state. The alloy potential is obtained from the corresponding elemental potential in the following empirical way (Raju et al 1996b):

$$
V(r)=V^{0}(r)\left[2\left(r^{0} / r\right)^{12}-\left(r^{0} / r\right)^{6}\right]
$$

where $r^{0}$ and $r$ refer to bond lengths in the elemental and alloyed state. Further $V_{i j}(r)$, the interaction potential between unlike atoms is obtained by taking the geometric mean of the respective $i$ and $j$ potentials, i.e.,

$$
V_{i j}=\sqrt{V_{i i} * V_{j j}} \text {. }
$$

Finally, an effective ordering potential for TiAl can be defined as (Raju et al 1996b)

$$
V_{\text {ord }}=\left[V_{\mathrm{Ti}-\mathrm{Al}}=-\frac{V_{\mathrm{Ti}-\mathrm{Ti}}+V_{\mathrm{Al}-\mathrm{Al}}}{2}\right] \text {. }
$$

In figure 3, the ordering potential for equiatomic TiAl is plotted. From the sign of $V_{\text {ord }}$ at the observed nn separation distance it is evident, that ordering is definitely favoured in TiAl. Using this calculated potential, the concentration of thermal 


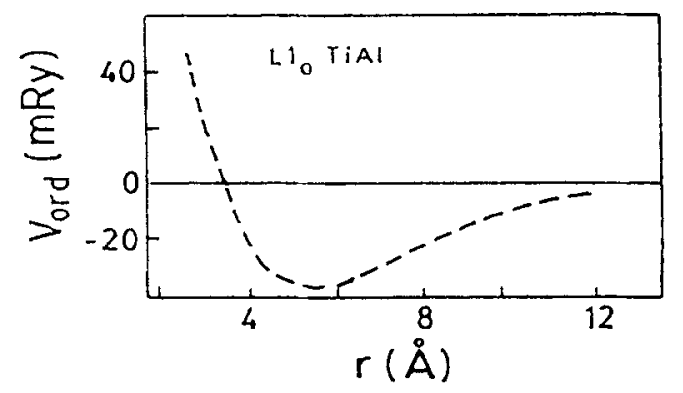

Figure 3. Calculated ordering potential for TiAl.

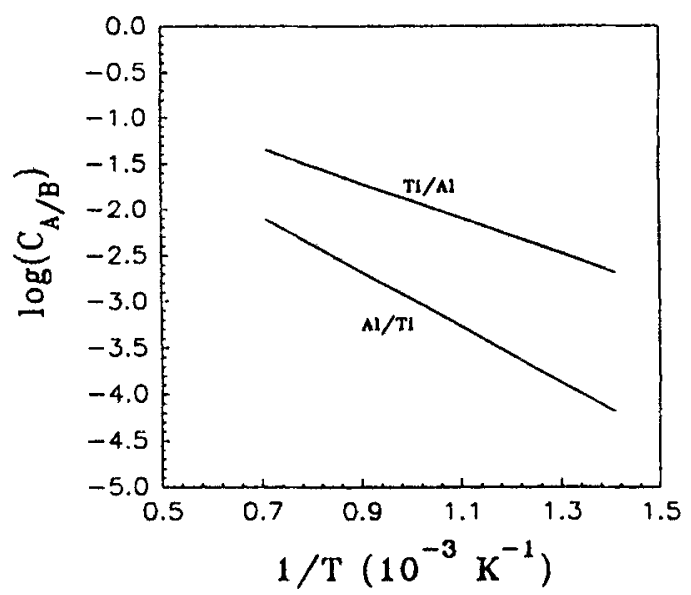

Figure 4. Calculated total thermal vacancy concentration plotted as a function of temperature.

vacancies is calculated within the spirit of a simple quasichemical model restricted to only $\mathrm{nn}$ interactions. The result is given in figure 4 . Although vibrational contributions to the total vacancy formation enthalpy have not been taken into account, the calculated value of point defect enthalpy of about $\sim 1.3 \mathrm{eV}$ agrees well with the reported experimental values of $\sim 1.4 \mathrm{eV}$ (Brossman et al 1994).

\section{Conclusions}

A modified WHM has been developed for possible applications in elucidating structural and thermophysical properties of transition metal and their alloys. A preliminary application of this potential to estimate the point defect properties of $\gamma$-TiAl is encouraging.

\section{Acknowledgements}

The authors thank Shri J B Gnanamoorthy and Dr Baldev Raj for their encouragement during the course of this work. Thanks are also due to Shri S Kanmani for many useful discussions. 


\section{References}

Ashcroft N W and Mermin N D 1976 Solid state physics (Tokyo: Holt-Saunders Int. Ed.)

Brossman U, Wurschunm R, Badura K and Schaefer H E 1994 Phys. Rev. B49 6457

Carlsson A E 1990 Solid State Phys, 431

de Hosson J Th M 1981 in Interatomic potentials and lattice defects (ed.) J K Lee (Warrendale, PA: Met. Soc. AIME)

Gehlen P C, Beeler J R and Jaffee R I (eds) 1972 Interatomic potentials and simulation of lattice defects (New York: Plenum)

Hafner J 1987 From Hamiltonian to phase diagrams (Berlin: Springer Ser. in Sol. St. Sci.) Vol. 70

Haglund J, Grimvall G and Jarlborg T 1993 Phys. Rev. B47 9279

Harrison W A 1970 Solid state theory (New York: MoGraw-Hill)

Harrison W A 1980 Electronic structure and properties of solids: The physics of chemical bond (San Fransisco: Freeman)

Harrison W A and Froyen S 1980 Phys. Rev. B21 3214

Harrison W A and Wills J M 1982 Phys. Rev. B25 5007

Pettifor D G 1983 in Physical metallurgy (eds) R W Cahn and P Haasen (Amsterdam: North-Holland)

Raju S, Mohandas E and Raghunathan V S 1996a Z. Metall. 8751

Raju S, Mohandas E and Raghunathan V S 1996b Scr. Metall. Mater. 34585

Vitek V and Srolovitz D J (eds) 1989 Atomistic simulation of materials: Beyond pair potentials (New York: Plenum)

Wills J M and Harrison W A 1983 Phys. Rev. 284363 